\title{
Prevalence of Kidney Stones in the United States
}

\author{
Charles D. Scales Jr. ${ }^{a,{ }^{*}}$, Alexandria C. Smith ${ }^{b}$, Janet M. Hanley ${ }^{b}$, Christopher S. Saigal ${ }^{c}$, and \\ Urologic Diseases in America Project \\ aUCLA Departments of Medicine and Urology, University of California, Los Angeles, Los Angeles, \\ CA, USA \\ bRAND Corporation, Santa Monica, CA, USA \\ 'Department of Urology, University of California, Los Angeles, Los Angeles, CA, USA; RAND \\ Corporation, Santa Monica, CA, USA
}

\begin{abstract}
Background-The last nationally representative assessment of kidney stone prevalence in the United States occurred in 1994. After a 13-yr hiatus, the National Health and Nutrition Examination Survey (NHANES) reinitiated data collection regarding kidney stone history.

Objective-Describe the current prevalence of stone disease in the United States, and identify factors associated with a history of kidney stones.
\end{abstract}

Design, setting, and participants-A cross-sectional analysis of responses to the 2007-2010 NHANES $(n=12110)$.

Outcome measurements and statistical analysis-Self-reported history of kidney stones. Percent prevalence was calculated and multivariable models were used to identify factors associated with a history of kidney stones.

Results and limitations-The prevalence of kidney stones was $8.8 \%$ (95\% confidence interval [CI], 8.1-9.5). Among men, the prevalence of stones was 10.6\% (95\% CI, 9.4-11.9), compared

\footnotetext{
"Corresponding author. Departments of Urology and Medicine, University of California, Los Angeles, 911 Broxton Avenue, 3rd Floor, Los Angeles, CA 90024, USA. Tel. +1 310794 2206; Fax: +1 310794 3288. cscales@ mednet.ucla.edu (C.D. Scales, Jr.).. Publisher's Disclaimer: This is a PDF file of an unedited manuscript that has been accepted for publication. As a service to our customers we are providing this early version of the manuscript. The manuscript will undergo copyediting, typesetting, and review of the resulting proof before it is published in its final citable form. Please note that during the production process errors may be discovered which could affect the content, and all legal disclaimers that apply to the journal pertain.

Author contributions: Charles D. Scales, Jr., had full access to all the data in the study and take responsibility for the integrity of the data and the accuracy of the data analysis.

Study concept and design: Scales, Saigal.

Acquisition of data: Smith, Hanley, Saigal.

Analysis and interpretation of data: Scales, Smith, Hanley, Saigal.

Drafting of the manuscript: Scales.

Critical revision of the manuscript for important intellectual content: Smith, Hanley, Saigal.

Statistical analysis: Scales, Smith, Hanley.

Obtaining funding: Saigal.

Administrative, technical, or material support: Smith, Hanley, Saigal.

Supervision: Saigal.

Other (specify): None.
}

Financial disclosures: Charles D. Scales, Jr., certifies that all conflicts of interest, including specific financial interests and relationships and affiliations relevant to the subject matter or materials discussed in the manuscript (eg, employment/ affiliation, grants or funding, consultancies, honoraria, stock ownership or options, expert testimony, royalties, or patents filed, received, or pending), are the following: Dr. Scales is supported by the Robert Wood Johnson Foundation Clinical Scholars program and the US Department of Veterans Affairs. 
with $7.1 \%$ (95\% CI, 6.4-7.8) among women. Kidney stones were more common among obese than normal-weight individuals (11.2\% [95\% CI, 10.0-12.3] compared with $6.1 \%$ [95\% CI, 4.87.4], respectively; $p<0.001)$. Black, non-Hispanic and Hispanic individuals were less likely to report a history of stone disease than were white, non-Hispanic individuals (black, non-Hispanic: odds ratio [OR]: 0.37 [95\% CI, 0.28-0.49], $p<0.001$; Hispanic: OR: 0.60 [95\% CI, 0.49-0.73], $p$ $<0.001)$. Obesity and diabetes were strongly associated with a history of kidney stones in multivariable models. The cross-sectional survey design limits causal inference regarding potential risk factors for kidney stones.

Conclusions-Kidney stones affect approximately 1 in 11 people in the United States. These data represent a marked increase in stone disease compared with the NHANES III cohort, particularly in black, non-Hispanic and Hispanic individuals. Diet and lifestyle factors likely play an important role in the changing epidemiology of kidney stones.

\section{Keywords}

Urinary lithiasis; Epidemiology; National Health and Nutrition Examination Survey

\section{Introduction}

Urinary stone disease is common and poses a significant health care burden in a workingage population. In 1994, data from the National Health and Nutrition Examination Survey (NHANES) estimated the prevalence of stone disease at $6.3 \%$ among men and $4.1 \%$ among women [1]. More recent analyses of claims data suggest rising utilization of health care resources for treating patients with stone disease [2,3]. However, there has been no nationally representative assessment of the prevalence of kidney stones in the United States since 1994. Thus, it remains unclear whether the claims data reflect changing patterns of care or changes in the epidemiology of kidney stones.

Recent investigations suggest that dietary and lifestyle factors play an important role in the risk of stone disease. Prospective data from the Health Professionals Follow-up Study and the Nurses' Health Study I and II note associations between incident stone disease and weight gain, body mass index (BMI), and diabetes mellitus [4,5]. These findings, combined with an obesity epidemic [6], support the hypothesis that the epidemiology of stone disease in the United States is changing.

Given this context, we sought to describe the current epidemiology of stone disease using a population-based cohort. We used data from the 2007-2010 NHANES sample to estimate the prevalence of stone disease in the United States. In the context of dramatically increasing obesity, we hypothesized that the prevalence of stone disease would be higher than $13 \mathrm{yr}$ previously, when these data were last queried by NHANES III.

\section{Methods}

\subsection{Data source}

We used data from the 2007-2010 NHANES. NHANES is a stratified, multistage probability survey of the noninstitutionalized US population. The institutional review board of RAND Corporation determined that the study design was exempted from the requirement for review.

\subsection{Study population}

The study population consisted of all participants responding to the questions regarding a history of kidney stones contained in the household survey component. These data were 
used to determine the population prevalence of stone disease. The participants in the medical examination component were used to identify risk factors associated with a history of kidney stones.

\subsection{Outcomes}

The primary outcome for the analysis was the response to the question, "Have you ever had kidney stones?" Since some stones may be incidental findings on abdominal imaging, our secondary outcome was based on participant response to the question, "How many times have you passed a kidney stone?" We considered any subject who reported passing at least one stone as having a history of symptomatic stone disease.

\subsection{Covariates}

Subject age and gender were used as reported in the public-use NHANES data. We used the racial and ethnic group variable reported by NHANES based on survey responses to questions about racial group and ethnicity. We combined responses from the NHANES categories of Mexican-American and Other Hispanic into a single Hispanic category according to analytic guidelines [7]. Otherwise, we used the racial/ethnic groups as reported by NHANES. We included household income as a measure of socioeconomic status, categorized as $\$ 0-19999, \$ 20000-34999, \$ 35000-74999$, and $\$ 75000$. A personal history of diabetes or gout was identified from the medical examination component, as was BMI, which was categorized as normal weight (18.0-24.9 kg/m²), overweight (25.0-29.9 $\left.\mathrm{kg} / \mathrm{m}^{2}\right)$, and obese $\left(\geq 30.0 \mathrm{~kg} / \mathrm{m}^{2}\right)$.

\subsection{Statistical analysis}

We calculated the percent prevalence of a self-reported history of stone disease, using weights and design variables as reported by NHANES to account for the complex survey structure and produce estimates representative of the total civilian, noninstitutionalized US population. We also adjusted prevalence estimates using data from the 1990 census to account for population changes since previous NHANES items regarding kidney stones were queried. Overall, changes in estimates because of population adjustment were small; therefore, we report unadjusted figures except where otherwise noted. A logistic regression model was constructed to identify factors associated with prevalent stone disease and account for the complex survey structure. We used SAS v.9.2 (Cary, NC, USA) for all analyses. Results were considered statistically significant with two-sided $a=0.05$.

\section{Results}

A total of 12110 participants responded to the question regarding a history of stone disease. The weighted overall prevalence of stone disease was $8.8 \%$ (95\% confidence interval [CI], 8.1-9.5). After population adjustment, the prevalence of stone disease was 8.4\% (95\% CI, 7.7-9.0). Men were more likely to report a history of stone disease than women (Table 1). Overall, estimates of the prevalence of kidney stones decreased only slightly after population adjustment (Table 2); therefore, we report unadjusted prevalence estimates unless otherwise specified. In men and women, the weighted prevalence increased with age; these effects were observed among all racial/ethnic groups (Table 3). Similar results were seen for the secondary outcome of symptomatic stone disease, although the point estimates were slightly lower (Table 1).

Variation in the prevalence of stone disease existed among different racial/ethnic groups (Table 3). The prevalence of stone disease was highest among non-Hispanic white individuals, at $10.3 \%$ (95\% CI, 9.4-11.1). Other ethnic/racial groups had a lower prevalence of stone disease. Among Hispanic individuals, the prevalence of kidney stones was $6.4 \%$ 
(95\% CI, 5.6-7.3), and among black, non-Hispanic individuals, the prevalence was $4.3 \%$

(95\% CI, 3.4-5.3). This variation persisted after population adjustment.

BMI was associated with a history of stone disease (Fig. 1). The prevalence of kidney stones was higher among obese individuals (11.2\% [95\% CI, 10.0-12.3]) and overweight individuals (9.2\% [95\% CI, 7.9-10.5]) than among individuals of normal weight $(6.1 \%$ [95\% CI, 4.8-7.2]) $(p<0.001)$. For males and females, respondents at normal weight had the lowest prevalence of stone disease (males: 7.8\% [95\% CI, 5.4-10.1]; females: $4.4 \%$ [95\% CI, 3.8-6.1]). Among obese males the prevalence of stone disease was $13.0 \%$ (95\% CI, 11.0-15.1), and among obese females the prevalence was 9.6\% (95\% CI, 8.3-10.8).

Several respondent characteristics were associated with a history of kidney stones in the multivariable model (Table 4). The odds of kidney stones in females was approximately two-thirds that in men (odds ratio [OR]: 0.63 [95\% CI, 0.52-0.74]; $p<0.001$ ). Individuals reporting their race/ethnicity as Hispanic or black, non-Hispanic were also less likely to report a history of stone disease (Hispanic OR: 0.60 [95\% CI, 0.49-0.73], $p<0.001$; black, non-Hispanic OR: 0.37 [95\% CI, 0.28-0.49], $p<0.001$ ).

Socioeconomic status was also associated with a history of kidney stones. Those individuals with an annual household income $\$ 19999$ were more likely than individuals earning $>\$ 75$ 000 annually to report a history of kidney stones (OR: 1.57 [95\% CI, 1.17-2.09], $p=0.002$ ). Individuals with an annual household income between $\$ 20000$ and $\$ 34999$ showed a similar increase in risk (OR: 1.65 [95\% CI, 1.27-2.15], $p<0.001)$ compared with the highincome group.

Conditions associated the metabolic syndrome were also associated with a reported history of stone disease (Table 4). The odds of kidney stones were 1.55 times higher among obese participants than among individuals of normal weight (OR: 1.55 [95\% CI, 1.25-1.94], $p<$ 0.001 ). Respondents with a history of diabetes were also more likely to report kidney stones (OR: 1.59 [95\% CI, 1.22-2.07], $p<0.001$ ). A statistically significant relationship among dietary intake variables and history of kidney stones was observed only for high levels of sodium intake (data not shown). For these reasons, we did not include dietary intake variables in the final model (Table 4).

\section{Discussion}

In the first NHANES assessment of kidney stone disease since 1994, we found that 1 in 11 persons in the United States reports a history of stone disease. Among the highest-risk groups, the prevalence of stone disease approached one in five persons. These findings represent a marked increase in the prevalence of stone disease over the intervening $15 \mathrm{yr}$ : In 1994, the reported prevalence of stone disease in the NHANES sample was only 5.2\% (ie, 1 in 20 persons) [1]. This dramatic increase in the prevalence of stone disease persisted even after adjusting for the changes in the US population since NHANES III.

The changing epidemiology of kidney stone disease was not limited to one specific demographic cohort; increasing prevalence of stones was noted in all age, gender, and racial/ ethnic groups, even on a population-adjusted basis. Among men, the overall prevalence of stone disease increased from $6.3 \%$ in $1988-1994$ to a population-adjusted $10.3 \%$ in $2007-$ 2010, representing a 63\% relative increase in the prevalence of stone disease [1]. The change in prevalence of kidney stones was particularly dramatic in black, non-Hispanics (a $1.7 \%$ prevalence in 1988-1994 [1] compared with a population-adjusted $4.5 \%$ prevalence in 2007-2010, representing a relative increase of $>150 \%$ ). The relative prevalence increase among Hispanic individuals was also dramatic. 
Recent claims-based analyses of health care utilization, particularly in the inpatient setting, have suggested a narrowing of the risk difference for stone disease between men and women $[2,3]$. Our data show that the overall prevalence of stone disease is $10.6 \%$ in males and $7.1 \%$ in females. At least two potential explanations exist for these apparently contradictory findings. First, these different findings could represent gender-based practice variation; that is, for reasons that are not illuminated by these data, men may be less likely to be treated as inpatients for stone disease. Alternately, our data are potentially consistent with the hypothesis that in the past, men were much more likely to form stones (represented by the large difference in prevalence between males and females aged $\geq 50 \mathrm{yr}$ ), but risks are currently more equal, as represented by the very similar prevalence of stone disease among males and females aged 20-49 yr. Because of the cross-sectional nature of the NHANES dataset, it is difficult to conclusively test this hypothesis. The NHANES dataset is specifically designed to estimate the prevalence of conditions in the US population and should be considered more reliable than claims-based utilization data for addressing questions of disease prevalence.

Prior analyses of NHANES data did not explore associations between markers of the metabolic syndrome (eg, overweight/obesity, diabetes) and the prevalence of stone disease. Since the last NHANES analysis, however, prospective data from three large cohorts clearly demonstrate an increasing risk of incident stone disease with obesity, weight gain, and other measures related to the metabolic syndrome [4,5]. Physiologically, obesity has been linked to increased renal excretion of calcium and uric acid, as well as increased urine acidity, all of which increase the risk of stone formation [8-13]. For these reasons, we investigated potential associations between obesity and a history of kidney stones. In multivariable models, we observed statistically significant relationships between a history of kidney stones and gout, diabetes, and obesity. Presuming obesity as a marker for the metabolic syndrome, which is linked epidemiologically and physiologically to risk of kidney stones, the epidemic of obesity in the United States is a likely explanation for the dramatic rise in the prevalence of stone disease. In our analysis, obesity was an equalizer between sexes; the prevalence of stone disease among obese women was higher than in normal-weight men. In addition, the rates of overweight/obesity are higher among black, non-Hispanic and Hispanic individuals than among white, non-Hispanic individuals [14]. These physiologic and epidemiologic data provide a plausible explanation for the observed changes in prevalence of stone disease in our sample compared with results from NHANES III [1].

Other factors may also contribute to the observed changes in stone disease prevalence. Mean average temperature is believed to be a major contributor to variation in geographic risk for stone disease $[15,16]$. Data from military deployments suggest that the onset of temperaturerelated changes in stone risk may occur within $90 \mathrm{~d}$ [17-20]. For these reasons, Brikowski and colleagues [21] speculate that temperature-related changes have contributed to, and may continue to exacerbate, the rising burden of stone disease.

Our findings should be considered in the context of important limitations. The rapid increase in radiologic imaging in the United States could translate into an increased diagnosis of asymptomatic kidney stones. To examine this possibility, we analyzed self-report of kidney stone passage as a secondary outcome. While the prevalence of stone passage was slightly lower than self-report of a history of kidney stones, these estimates still represent a marked increase in stone disease. The prevalence of stone disease increases with age; therefore, the aging of the US population would be expected to contribute to an overall increase in the prevalence of stone disease absent any other influences. However, population adjustment of our estimates suggested that changes in prevalence because of the aging of the population only partially explained the observed changes in prevalence. 
Finally, our results should be considered in the context of the cross-sectional design of the NHANES instrument. While we explored nutritional intake (including moisture) in our multivariable models, we did not find important associations with a history of stone disease. This finding is consistent with the findings from the NHANES III analysis, in which significant associations between dietary factors and stone history were not observed [1]. Since the temporal relationship of participant diet at the time of NHANES and a past history of stone disease is unclear, it is not surprising that prospectively identified associations between diet and incident stone disease [4,5] were not observed in this cross-sectional design.

These limitations notwithstanding, our findings have important implications for treating patients with kidney stones in the United States. Continued increases in prevalence will presumably sustain recent increases in health care expenditures for treating patients with stone disease [2,22]. Currently, patient treatment is characterized primarily by surgical intervention for symptomatic stone events rather than management of the chronic metabolic condition that leads to recurrence in $\mathbf{5 0 \%}$ of patients within $5 \mathrm{yr}$ in a primarily working-age population [23]. Evidence from randomized controlled trials suggests that lifestyle and pharmacologic interventions are effective for secondary prevention of kidney stones [2427]. The rapidly changing prevalence of kidney stones suggests that further efforts toward secondary prevention may be indicated.

\section{Conclusions}

The prevalence of kidney stones in the United States is markedly higher than when last measured in 1988-1994, and this increase is likely related to dietary and lifestyle factors. Given the temporary disability imposed by a symptomatic stone event, these findings have important implications for a disease that burdens a primarily working-age population. These findings suggest that the direct and indirect costs of kidney stones will continue to rise in the United States, and efforts should be directed toward ameliorating the burden of urinary stone disease.

\section{Acknowledgments}

Funding/Support and role of the sponsor: This analysis was funded by grant N01-DK70003 from the National Institute of Diabetes and Digestive and Kidney Diseases. The National Institute of Diabetes and Digestive and Kidney Diseases had no role in the design or conduct of the study; collection, management, analysis, or interpretation of the data; or preparation, review, or approval of the manuscript. The views expressed in this article are those of the authors and do not necessarily represent the official view of the National Institute of Diabetes and Digestive and Kidney Diseases or the US Department of Veterans Affairs.

\section{References}

1. Stamatelou KK, Francis ME, Jones CA, Nyberg LM, Curhan GC. Time trends in reported prevalence of kidney stones in the United States: 1976-1994. Kidney Int. 2003; 63:1817-23. [PubMed: 12675858]

2. Pearle MS, Calhoun EA, Curhan GC. Urologic Diseases in America project: urolithiasis. J Urol. 2005; 173:848-57. [PubMed: 15711292]

3. Scales CD Jr, Curtis LH, Norris RD. Changing gender prevalence of stone disease. J Urol. 2007; 177:979-82. [PubMed: 17296391]

4. Taylor EN, Stampfer MJ, Curhan GC. Obesity, weight gain, and the risk of kidney stones. JAMA. 2005; 293:455-62. [PubMed: 15671430]

5. Taylor EN, Stampfer MJ, Curhan GC. Diabetes mellitus and the risk of nephrolithiasis. Kidney Int. 2005; 68:1230-5. [PubMed: 16105055] 
6. Hedley AA, Ogden CL, Johnson CL, Carroll MD, Curtin LR, Flegal KM. Prevalence of overweight and obesity among US children, adolescents, and adults, 1999-2002. JAMA. 2004; 291:2847-50. [PubMed: 15199035]

7. Analytic note regarding 2007-2010 survey design changes and combining data across other survey cycles. US Centers for Disease Control and Prevention Web site. http://www.cdc.gov/nchs/data/nhanes/analyticnote_2007-2010.pdf

8. Ekeruo WO, Tan YH, Young MD. Metabolic risk factors and the impact of medical therapy on the management of nephrolithiasis in obese patients. J Urol. 2004; 172:159-63. [PubMed: 15201761]

9. Maalouf NM, Sakhaee K, Parks JH, Coe FL, Adams-Huet B, Pak CY. Association of urinary pH with body weight in nephrolithiasis. Kidney Int. 2004; 65:1422-5. [PubMed: 15086484]

10. Powell CR, Stoller ML, Schwartz BF. Impact of body weight on urinary electrolytes in urinary stone formers. Urology. 2000; 55:825-30. [PubMed: 10840085]

11. Rumenapf G, Schmidtler J, Schwille PO. Intestinal calcium absorption during hyperinsulinemic euglycemic glucose clamp in healthy humans. Calcif Tissue Int. 1990; 46:73-9. [PubMed: 2105152]

12. Kerstetter J, Caballero B, O'Brien K, Wurtman R, Allen L. Mineral homeostasis in obesity: effects of euglycemic hyperinsulinemia. Metabolism. 1991; 40:707-13. [PubMed: 1870424]

13. Abate N, Chandalia M, Cabo-Chan AV Jr, Moe OW, Sakhaee K. The metabolic syndrome and uric acid nephrolithiasis: novel features of renal manifestation of insulin resistance. Kidney Int. 2004; 65:386-92. [PubMed: 14717908]

14. Flegal KM, Carroll MD, Ogden CL, Curtin LR. Prevalence and trends in obesity among US adults, 1999-2008. JAMA. 2010; 303:235-41. [PubMed: 20071471]

15. Soucie JM, Coates RJ, McClellan W, Austin H, Thun M. Relation between geographic variability in kidney stones prevalence and risk factors for stones. Am J Epidemiol. 1996; 143:487-95. [PubMed: 8610664]

16. Chen YY, Roseman JM, Devivo MJ, Huang CT. Geographic variation and environmental risk factors for the incidence of initial kidney stones in patients with spinal cord injury. J Urol. 2000; 164:21-6. [PubMed: 10840416]

17. Clark JY. Renal calculi in army aviators. Aviat Space Environ Med. 1990; 61:744-7. [PubMed: 2400380]

18. Cramer JS, Forrest K. Renal lithiasis: addressing the risks of austere desert deployments. Aviat Space Environ Med. 2006; 77:649-53. [PubMed: 16780245]

19. Pierce LW, Bloom B. Observations on urolithiasis among American troops in a desert area. J Urol. 1945; 54:466-70. [PubMed: 21005411]

20. Evans K, Costabile RA. Time to development of symptomatic urinary calculi in a high risk environment. J Urol. 2005; 173:858-61. [PubMed: 15711293]

21. Brikowski TH, Lotan Y, Pearle MS. Climate-related increase in the prevalence of urolithiasis in the United States. Proc Natl Acad Sci U S A. 2008; 105:9841-6. [PubMed: 18626008]

22. Litwin, MS.; Saigal, CS. Introduction. US Department of Health and Human Services, Public Health Service, National Institutes of Health, National Institute of Diabetes and Digestive and Kidney Diseases; Washington, DC: 2007.

23. Saigal CS, Joyce G, Timilsina AR. Direct and indirect costs of nephrolithiasis in an employed population: opportunity for disease management? Kidney Int. 2005; 68:1808-14. [PubMed: 16164658]

24. Pearle MS, Roehrborn CG, Pak CY. Meta-analysis of randomized trials for medical prevention of calcium oxalate nephrolithiasis. J Endourol. 1999; 13:679-85. [PubMed: 10608521]

25. Escribano J, Balaguer A, Pagone F, Feliu A, Roque IFM. Pharmacological interventions for preventing complications in idiopathic hypercalciuria. Cochrane Database Syst Rev. 2009:CD004754. [PubMed: 19160242]

26. Fink HA, Akornor JW, Garimella PS. Diet, fluid, or supplements for secondary prevention of nephrolithiasis: a systematic review and meta-analysis of randomized trials. Eur Urol. 2009; 56:72-80. [PubMed: 19321253]

27. Qiang W, Ke Z. Water for preventing urinary calculi. Cochrane Database Syst Rev. 2004:CD004292. [PubMed: 15266525] 


\section{Take-home message}

The prevalence of kidney stones in the United States has increased from 1 in 20 persons to 1 in 11 persons since 1994. This change is likely related to the rising prevalence of the metabolic syndrome in the US population. 


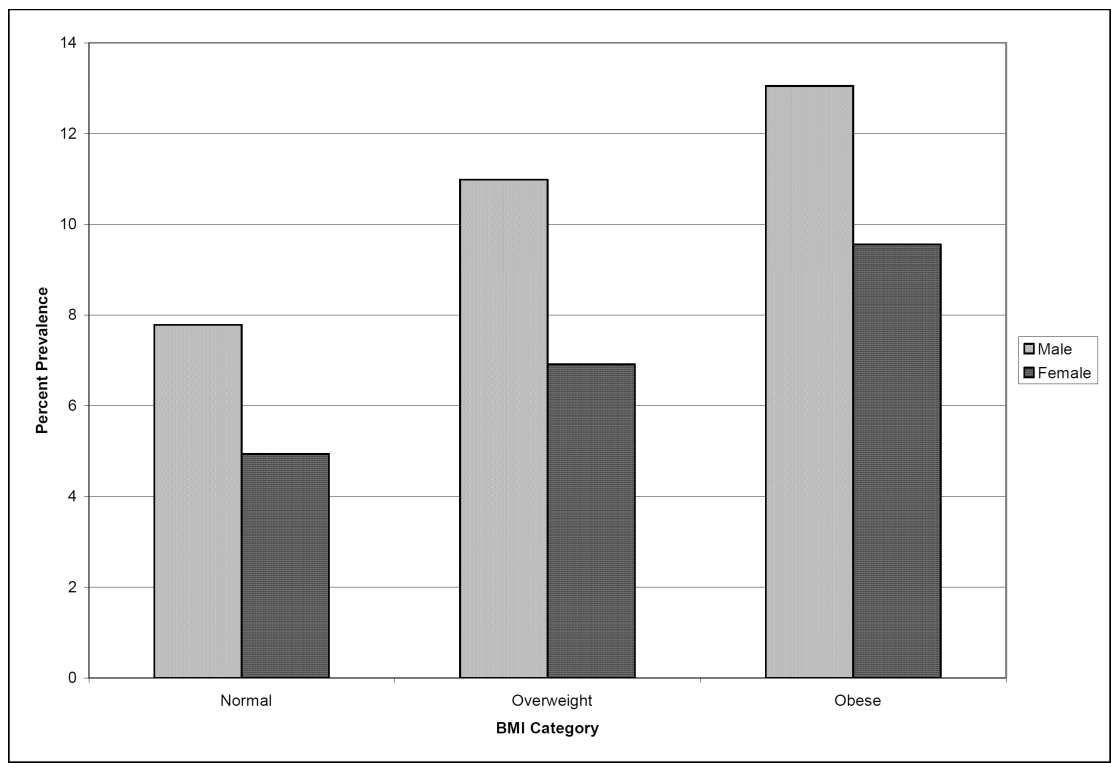

Fig. 1.

Weighted prevalence of stone disease by gender and body mass index (BMI) category. 


\section{Table 1}

Weighted (unadjusted) percent prevalence of stone disease by population characteristic

\begin{tabular}{|c|c|c|c|c|}
\hline \multirow[b]{2}{*}{ Characteristic } & \multicolumn{2}{|c|}{ History of kidney stones, \% (95\% CI) } & \multicolumn{2}{|c|}{ History of passing at least one kidney stone, $\%(95 \% \mathrm{CI})$} \\
\hline & Male & Female & Male & Female \\
\hline \multicolumn{5}{|l|}{ Age group, yr } \\
\hline $20-29$ & $3.4(2.1-4.7)$ & $3.4(2.2-4.7)$ & $3.3(2.0-4.5)$ & $2.5(1.3-3.7)$ \\
\hline $30-39$ & $6.9(5.0-8.8)$ & $5.9(4.5-7.2)$ & $6.5(4.6-8.5)$ & $5.0(3.5-6.4)$ \\
\hline $40-49$ & $9.8(7.3-12.3)$ & $7.6(5.6-9.5)$ & $8.1(5.9-10.4)$ & $6.4(4.7-8.1)$ \\
\hline $50-59$ & $13.1(10.3-15.9)$ & $8.1(5.9-10.3)$ & $11.1(13.4-19.3)$ & $6.9(4.8-9.0)$ \\
\hline $60-69$ & $19.1(15.9-22.4)$ & $9.4(6.6-12.2)$ & $16.3(13.4-19.3)$ & $8.4(5.6-11.3)$ \\
\hline $70+$ & $18.8(16.5-21.0)$ & $9.4(7.5-11.3)$ & $16.0(13.8-18.3)$ & $7.1(5.5-8.8)$ \\
\hline All ages & $10.6(9.4-11.9)$ & $7.1(6.4-7.8)$ & $9.2(8.1-10.3)$ & $5.9(5.2-6.6)$ \\
\hline
\end{tabular}

$\mathrm{CI}=$ confidence interval. 
Table 2

Unadjusted and population-adjusted percent prevalence of stone disease by population characteristic

\begin{tabular}{|lrrrr|}
\hline & \multicolumn{2}{c}{ History of kidney stones, males } & \multicolumn{2}{c|}{ History of kidney stones, females } \\
Characteristic & Unadjusted, \% (95\% CI) & Adjusted, \% (95\% CI) & Unadjusted, \% (95\% CI) & Adjusted, \% (95\% CI) \\
\hline All groups & $10.6(9.4-11.9)$ & $10.3(9.2-11.3)$ & $7.1(6.4-7.8)$ & $6.7(6.1-7.4)$ \\
Non-Hispanic, white & $12.8(11.3-14.3)$ & $11.8(10.4-13.2)$ & $7.9(7.0-8.8)$ & $7.5(6.7-8.4)$ \\
Hispanic & $7.1(5.7-8.4)$ & $8.8(7.4-10.2)$ & $5.7(4.6-6.9)$ & $6.1(4.9-7.3)$ \\
Non-Hispanic, black & $4.5(3.4-5.6)$ & $4.8(3.7-5.9)$ & $4.2(2.7-5.7)$ & $4.2(2.8-5.6)$ \\
Other race/multiracial & $5.6(2.5-8.8)$ & $5.3(2.2-8.5)$ & $6.1(2.7-9.6)$ & $5.6(2.4-8.8)$ \\
\hline
\end{tabular}

$\mathrm{CI}=$ confidence interval 
Table 4

Multivariable regression model predicting history of kidney stones

\begin{tabular}{|c|c|c|}
\hline Characteristic & Odds ratio $(95 \%$ CI $)$ & $p$ value \\
\hline \multicolumn{3}{|l|}{ Age, yr } \\
\hline $20-39$ & 1.00 (referent) & - \\
\hline $40-59$ & $1.83(1.37-2.45)$ & $<0.001$ \\
\hline$\lcm{60}$ & $2.18(1.74-2.73)$ & $<0.001$ \\
\hline Female & $0.63(0.52-0.75)$ & $<0.001$ \\
\hline \multicolumn{3}{|l|}{ Race } \\
\hline White, non-Hispanic & 1.00 (referent) & - \\
\hline Black, non-Hispanic & $0.37(0.28-0.49)$ & $<0.001$ \\
\hline Hispanic & $0.60(0.49-0.73)$ & $<0.001$ \\
\hline Other/multiracial & $0.57(0.37-0.89)$ & 0.014 \\
\hline \multicolumn{3}{|l|}{ BMI category } \\
\hline Normal & 1.00 (referent) & - \\
\hline Overweight & $1.29(0.96-1.72)$ & 0.0875 \\
\hline Obese & $1.55(1.25-1.94)$ & $<0.001$ \\
\hline \multicolumn{3}{|l|}{ Household income, \$ } \\
\hline$\geq 75000$ & 1.00 (referent) & - \\
\hline $35000-74999$ & $1.49(1.16-1.92)$ & 0.002 \\
\hline $20000-34999$ & $1.65(1.27-2.15)$ & $<0.001$ \\
\hline 0-19999 & $1.57(1.17-2.09)$ & 0.002 \\
\hline Diabetes & $1.59(1.22-2.07)$ & $<0.001$ \\
\hline Gout & $1.92(1.44-2.56)$ & $<0.001$ \\
\hline
\end{tabular}

$\mathrm{CI}=$ confidence interval BMI $=$ body mass index. 